\title{
CONVERGENCE- AND SUM-FACTORS FOR SERIES OF COMPLEX NUMBERS
}

\author{
BY
}

\author{
E. CALABI AND A. DVORETZKY
}

\section{Introduction.}

1.1. Let $\sum_{n=1}^{\infty} a_{n}$ be a series of real numbers tending to zero, $\zeta^{(1)} \geqq 0$ and $\zeta^{(2)} \leqq 0$. Then it is obvious that a sequence $\zeta_{1}, \cdots, \zeta_{n}, \cdots$ with each $\zeta_{n}$ equal to either $\zeta^{(1)}$ or $\zeta^{(2)}$ may be chosen so that $\sum_{n=1}^{\infty} \zeta_{n} a_{n}$ be convergent. If, furthermore, $\sum\left|a_{n}\right|$ is divergent and $\zeta^{(1)} \zeta^{(2)} \neq 0$, the sequence $\left(\zeta_{n}\right)$ may be chosen so that $\sum_{n=1}^{\infty} \zeta_{n} a_{n}$ converges to any preassigned sum.

On passing from series of real numbers to series of complex ones, the second statement above clearly ceases to be true while the first one, though remaining valid (cf. below), is far from obvious. It is the primary object of the present paper to extend and generalize the above and related statements to series of complex numbers.

1.2. The quotation of the principal results will be facilitated if the following definitions are introduced.

Definition 1. A set $\mathrm{Z}$ of complex numbers is called a convergence factor set if, given any sequence $\left(a_{n}\right)_{1}^{\infty}$ of complex numbers satisfying

$$
\lim _{n=\infty} a_{n}=0
$$

there exists a sequence $\left(\zeta_{n}\right)_{1}^{\infty}$ with $\zeta_{n} \in \mathrm{Z}(n=1,2, \cdots)$ for which $\sum \zeta_{n} a_{n}$ is convergent.

Definition 2. A set $\mathrm{Z}$ of complex numbers is called a sum factor set if, given any sequence $\left(a_{n}\right)_{1}^{\infty}$ of complex numbers satisfying (1) and

$$
\sum_{n=1}^{\infty}\left|a_{n}\right|=\infty
$$

and any complex number $s$, there exists a sequence $\left(\zeta_{n}\right)_{1}^{\infty}$, with $\zeta_{n} \in \mathrm{Z}$ $(n=1,2, \cdots)$ for which $\sum_{n=1}^{\infty} \zeta_{n} a_{n}$ converges to $s$.

The following two theorems give a simple characterization of bounded convergence and sum factor sets.

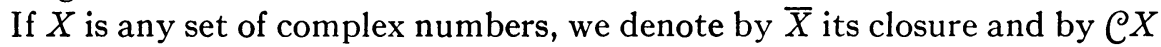
its convex hull, that is, the (not necessarily closed) intersection of all convex sets containing $X$.

THEOREM 1. A bounded set $\mathrm{Z}$ is a convergence factor set if and only if $0 \in \overline{\mathrm{CZ}}$.

THEOREM 2. A bounded set $\mathrm{Z}$ is a sum factor set if and only if 0 is an interior

Presented to the Society, December 28, 1948; received by the editors June 1, 1949. 
point of $\mathrm{CZ}$.

The only cases of convergence factor sets $\mathrm{Z}$ known before seem to be those whose closure $\overline{\mathrm{Z}}$ contains a pair of points $(\zeta$ and $-\zeta)$ symmetric about the origin. Even this is (for $\zeta \neq 0$ ) not trivial and due to Ch. Hanani and one of the present authors who proved $[1,3]\left(^{1}\right)$ that if $\sum a_{n}$ is any series of complex numbers tending to zero it is possible to change the signs so that the resulting series $\sum \pm a_{n}$ is convergent, that is, that $\{1,-1\}$ (or $\{\zeta,-\zeta\}$ ) is a convergence factor set.

The origin can be an interior point of $\mathcal{C Z}$ only if it is an interior point of a nondegenerate triangle or a nondegenerate quadrilateral with vertices in $\mathrm{Z}$. Thus if a set $\mathbf{Z}$ is a sum factor set it necessarily contains a subset of three or four points having the same property. The only previously published instances of sum factor sets seem to be due to H. Hornich who proved [4] that for $k \geqq 3$ the set of $k$ th roots of unity is a sum factor set.

1.3. The proof of Theorem 2 is straightforward but that of Theorem 1 is more tricky. Dvoretzky and Hanani [3] make some use of the group property of sign changes that if the signs of some terms in $\sum a_{n}$ are changed, and then a similar operation is applied to the resulting series, the final series is again of the form $\sum \pm a_{n}$. No similar property obtains if $\{1,-2\}$, say, are used as multipliers instead of $\{1,-1\}$. In order to overcome this difficulty it was found convenient to obtain Theorem 1 as a special case of a more general result relating to the case when the factors $\zeta_{n}$ instead of being taken from a fixed set $\mathrm{Z}$ are taken from different sets $\mathbf{Z}_{n}$.

Definition 3. A sequence $\left(\mathbf{Z}_{n}\right)_{1}^{\infty}$ of sets of complex numbers is called a convergence factor sequence if, given any sequence of complex numbers $\left(a_{n}\right)_{1}^{\infty}$ satisfying (1), there exists a sequence $\left(\zeta_{n}\right)_{1}^{\infty}$ with $\zeta_{n} \in \mathrm{Z}_{n}(n=1,2, \cdots)$ for which $\sum \zeta_{n} a_{n}$ is convergent.

In $\$ 2$ we prove

TheOREm 3. A sequence $\left(\mathrm{Z}_{n}\right)_{1}^{\infty}$ of uniformly bounded sets of complex numbers is a convergence factor sequence if and only if $\sum_{n=1}^{\infty} d_{n}<\infty$, where

$$
d_{n}=\inf _{\zeta \in \mathcal{C} \mathrm{z}_{n}}|\zeta|=\min _{\zeta \in \overline{\mathcal{C}_{\mathrm{z}_{n}}}}|\zeta|
$$

is the distance of the origin from the convex hull of $\mathrm{Z}_{n}$.

If all $\mathbf{Z}_{n}$ are identical, so are all $d_{n}$ and the above condition reduces to the requirement that the common value of all $d_{n}$ be zero. Thus, Theorem 3 specializes to Theorem 1 in this particular case.

In $\$ 3$ a similar extension of Theorem 2 is given. We introduce the following definition.

Definition 4. A sequence $\left(\mathrm{Z}_{n}\right)_{1}^{\infty}$ of sets of complex numbers is called a sum

(1) Numbers in brackets refer to the bibliography at the end of the paper. 
factor sequence if, given any sequence of complex numbers $\left(a_{n}\right)_{1}^{\infty}$ satisfying (1) and (2), and a complex number $s$, there exists a sequence $\left(\zeta_{n}\right)_{1}^{\infty}$ with $\zeta_{n} \in \mathrm{Z}_{n}$ $(n=1,2, \cdots)$ for which $\sum_{n=1}^{\infty} \zeta_{n} a_{n}$ converges to $s$.

We obtain

Theorem 4. A sequence $\left(\mathrm{Z}_{n}\right)_{1}^{\infty}$ of uniformly bounded nonempty sets of complex numbers is a sum factor sequence if and only if there exist an integer $N$ and $a$ positive $\delta$ such that

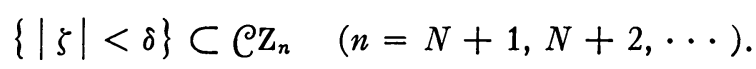

Thus $\left(Z_{n}\right)$ is a sum factor sequence if and only if 0 is an interior point of the intersection of all but a finite number of $C Z_{n}$. Theorem 2 is clearly a special case.

1.4. In $\$ 4$ we show (Proposition 4 ) that a set $S$ of complex numbers is the set of limit points of the partial sums of some series $\sum_{n=1}^{\infty} a_{n}$ satisfying (1) if and only if it is a continuum or becomes one on adjoining the point at infinity (cf. also Proposition 5). We then prove (Theorem 5) a result which shows in particular that if $S$ satisfies the above requirements, if $\left(\mathrm{Z}_{n}\right)$ satisfies the conditions of Theorem 4 , and if $\left(a_{n}\right)$ satisfies (1) and (2), then $\zeta_{n} \in \mathbf{Z}_{n}$ may be chosen so that $S$ coincides with the set of limit points of the partial sums of $\sum_{n=1}^{\infty} \zeta_{n} a_{n}$.

Thus a uniformly bounded sequence of sum factors can secure not only convergence to a preassigned sum, but also the oscillation of the partial sums through any set which can at all be a derived set of partial sums of a series satisfying (1). For the special case when all $\mathbf{Z}_{n}$ coincide with the set of $k$ th roots of unity ( $k \geqq 3$ fixed) a partial result of the same kind was obtained by Hornich [5].

In $\$ 5$ we deal with nonbounded convergence and sum factor sets and sequences. A complete and easy characterization of convergence factor sets and sequences is obtained (Theorems 6 and 7) but we did not obtain a similar complete solution of the corresponding problem for sum factor sequences (sum factor sets are characterized by Theorem 8 ).

In $\$ 6$ an application to series of functions is given and $\$ 7$ contains a remark on the value of a constant encountered in $\$ 2$.

1.5. In conclusion we note that many of the results of this paper extend also to vectors in any Euclidean space and more general linear spaces. In particular, if we confine ourselves to real (scalar) factors hardly any change in the proofs is required in order to establish the analogues of Theorem 1 and 3 for arbitrary finite-dimensional Banach spaces. (For infinitely-dimensional spaces, even for Hilbert spaces, even Theorem 1 with $\mathrm{Z}=\{1,-1\}$ obviously fails.)

\section{Convergence factors.}

2.1. In studying convergence factors we find it convenient to refer to the following 
LеммA. If a segment in the plane of length less than or equal to 2 is at a distance less than or equal to 1 from the origin, then either at least one of its points is at a distance less than or equal to 1 from the origin or both end points are at a distance less than $5^{1 / 2}$ from it.

The verification of the lemma being immediate, we proceed to apply it and prove the following propositions.

Proposition 1. Let $\left\{\alpha_{n}, \beta_{n}, \gamma_{n}\right\}(n=1,2, \cdots)$ be a sequence of triplets of (not necessarily distinct) complex numbers satisfying

$$
\max \left(\left|\beta_{n}-\alpha_{n}\right|,\left|\gamma_{n}-\beta_{n}\right|,\left|\alpha_{n}-\gamma_{n}\right|\right) \leqq 2 \quad(n=1,2, \cdots)
$$

and

$$
0 \in \mathcal{C}\left\{\alpha_{n}, \beta_{n}, \gamma_{n}\right\} \quad(n=1,2, \cdots) .
$$

Then, given a positive integer $N$, there exists a sequence $\left(\zeta_{n}\right)_{n=1}^{N}$ with $\zeta_{n} \in\left\{\alpha_{n}\right.$, $\left.\beta_{n}, \gamma_{n}\right\}$ which satisfies

$$
\left|\sum_{\nu=1}^{n} \zeta_{\nu}\right|<5^{1 / 2} \quad(n=1,2, \cdots, N) .
$$

In order to establish this proposition we prove the somewhat stronger

Proposition 2. Under the assumptions of Proposition 1 the following conclusion holds: Given a positive integer $N$, there exist an integer $u(N)$ and two sequences $\left(\zeta_{n, N}^{\prime}\right)_{n=1}^{N}$ and $\left(\zeta_{n, N}^{\prime \prime}\right)_{n=1}^{N}$ satisfying

$$
\begin{array}{rr}
1 \leqq u(N) \leqq N & \\
\left\{\zeta_{n, N}^{\prime}, \zeta_{n, N}^{\prime \prime}\right\} \subset\left\{\alpha_{n}, \beta_{n}, \gamma_{n}\right\} & (n=1,2, \cdots, N), \\
\zeta_{n, N}^{\prime}=\zeta_{n, N}^{\prime \prime} & \text { for } n \neq u(N), \\
\left|\sum_{\nu=1}^{n} \zeta_{\nu, N}^{\prime}\right|<5^{1 / 2} \text { and }\left|\sum_{\nu=1}^{n} \zeta_{\nu, N}^{\prime \prime}\right|<5^{1 / 2} & (n=1,2, \cdots, N-1),
\end{array}
$$

and

$$
\min _{0 \leqq \mu 1}\left|\mu \sum_{\nu=1}^{N} \zeta_{\nu, N}^{\prime}+(1-\mu) \sum_{\nu=1}^{N} \zeta_{\nu, N}^{\prime \prime}\right| \leqq 1 .
$$

Because of (7), (9), (10), and the lemma, Proposition 2 clearly implies Proposition 1.

2.2. Proof of Proposition 2. The proof proceeds by induction on $N$. $u(1)=1$ by (6). Since the triangle $\mathcal{C}\left\{\alpha_{1}, \beta_{1}, \gamma_{1}\right\}$ contains the origin, it meets the unit disc $|z| \leqq 1$ and thus, by (3), at least one of its sides meets the unit disc. Let $\zeta_{1,1}^{\prime}$ and $\zeta_{1,1}^{\prime \prime}$ denote the end points of this side, then all the contentions of Proposition 2 with $N=1$ hold.

Assume now the truth of Proposition 2 for some fixed $N \geqq 1$. Put 


$$
s_{n, N}^{\prime}=\sum_{\nu=1}^{n} \zeta_{\nu, N}^{\prime}, \quad s_{n, N}^{\prime \prime}=\sum_{\nu=1}^{n} \zeta_{\nu, N}^{\prime \prime} \quad(n=1,2, \cdots, N)
$$

and consider separately the mutually exclusive and jointly exhaustive cases $A_{1}, A_{2}, B_{1}, B_{2}$, and $B_{3}$.

Case $\mathrm{A}_{1} \cdot\left|s_{N, N}^{\prime}\right| \leqq 1$.

Put $u(N+1)=N+1$ and $\zeta_{n, N+1}^{\prime}=\zeta_{n, N+1}^{\prime \prime}=\zeta_{n, N}^{\prime}(n=1,2, \cdots, N)$. These values satisfy (6) and (8) with $N$ replaced by $N+1$ and, by the induction assumption, also (9). Because of (4), the triangle( $\left.{ }^{2}\right) s_{N, N+1}^{\prime}+\mathcal{C}\left\{\alpha_{N+1}, \beta_{N+1}, \gamma_{N+1}\right\}$ contains the point $s_{N, N+1}^{\prime}=s_{N, N}^{\prime}$ which is assumed to lie within the unit disc. By (3) a side of this triangle meets the unit disc. Choose $\zeta_{N+1, N+1}^{\prime}$ and $\zeta_{N+1, N+1}^{\prime \prime}$ from among $\alpha_{N+1}, \beta_{N+1}$, and $\gamma_{N+1}$ so that $s_{N, N+1}^{\prime}+\zeta_{N+1, N+1}^{\prime}$ and $s_{N, N+1}^{\prime}+\zeta_{N+1, N+1}^{\prime \prime}$ be the end points of such a side. Then the sequences $\left(\zeta_{n, N+1}^{\prime}\right)$, $\left(\zeta_{n, N+1}^{\prime \prime}\right)(n=1,2, \cdots, N+1)$ satisfy $(7)$ and (10) with $N$ replaced by $N+1$. Thus, in this case, Proposition 2 holds for $N+1$.

Case $\mathrm{A}_{2 \cdot}\left|s_{N, N}^{\prime}\right|>1$ but $\left|s_{N, N}^{\prime \prime}\right| \leqq 1$.

This case reduces to the preceding one on interchanging the superscripts 'and $"$.

Case B. $\left|s_{N, N}^{\prime}\right|>1$ and $\left|s_{N, N}^{\prime \prime}\right|>1$.

It follows from (3), (7), (8), (10), and the lemma that

$$
\max \left(\left|s_{N, N}^{\prime}\right|,\left|s_{N, N}^{\prime \prime}\right|\right)<5^{1 / 2} \text {. }
$$

Consider the set of six (not necessarily distinct) points

$$
\left\{s_{N, N}^{\prime}, s_{N, N}^{\prime \prime}\right\}+\left\{\alpha_{N+1}, \beta_{N+1}, \gamma_{N+1}\right\}
$$

and its convex hull

$$
P_{N}=\mathcal{C}\left\{s_{N, N}^{\prime}, s_{N, N}^{\prime \prime}\right\}+\mathcal{C}\left\{\alpha_{N+1}, \beta_{N+1}, \gamma_{N+1}\right\} .
$$

$P_{N}$ is the union of two triangles and three parallelograms, namely

$$
\begin{aligned}
P_{n}= & \left(s_{N, N}^{\prime}+\mathcal{C}\left\{\alpha_{N+1}, \beta_{N+1}, \gamma_{N+1}\right\}\right) \cup\left(s_{N, N}^{\prime \prime}+\mathcal{C}\left\{\alpha_{N+1}, \beta_{N+1}, \gamma_{N+1}\right\}\right) \\
& \cup\left(\mathcal{C}\left\{s_{N, N}^{\prime}, s_{N, N}^{\prime \prime}\right\}+\mathcal{C}\left\{\beta_{N+1}, \gamma_{N+1}\right\}\right) \\
& \cup\left(\mathcal{C}\left\{s_{N, N}^{\prime}, s_{N, N}^{\prime \prime}\right\}+\mathcal{C}\left\{\gamma_{N+1}, \alpha_{N+1}\right\}\right) \\
& \cup\left(\mathcal{C}\left\{s_{N, N}^{\prime}, s_{N, N}^{\prime \prime}\right\}+\mathcal{C}\left\{\alpha_{N+1}, \beta_{N+1}\right\}\right) .
\end{aligned}
$$

By (4) the two triangles in (12) contain respectively $s_{N, N}^{\prime}$ and $s_{N, N}^{\prime \prime}$; since $P_{N}$ is convex, it contains the segment joining $s_{N, N}^{\prime}$ and $s_{N, N}^{\prime \prime}$, and hence, by (10), a point $s_{N}^{\circ}$ with $\left|s_{N}^{\circ}\right| \leqq 1$. Since $s_{N}^{\circ}$ must belong to one of the triangles or parallelograms of (12), this triangle or parallelogram meets the unit disc. Since no quadrangle all of whose sides are of length less than or equal to 2 can contain the unit circle in its interior, it follows that at least one of the

(2) Here and elsewhere in the paper, if $A$ and $B$ are sets of complex numbers we denote by $A+B$ their group theoretic sum, that is, the set of all $a+b$ with $a \in A, b \in B$. 
nine segments constituting the boundaries of the two triangles and three parallelograms of (12) meets the unit disc.

Let these nine segments be arranged in some order and let $\sigma_{N}$ be the first of these which meets the unit disc; we now distinguish three cases:

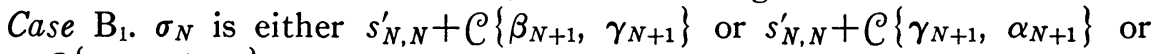
$s_{N, N}^{\prime}+\mathcal{C}\left\{\alpha_{N+1}, \beta_{N+1}\right\}$.

In this case we set $u(N+1)=N+1, \zeta_{n, N+1}^{\prime}=\zeta_{n, N+1}^{\prime \prime}=\zeta_{n, N}^{\prime}(n=1,2, \cdots, N)$ and $\left\{\zeta_{N+1, N+1}^{\prime}, \zeta_{N+1, N+1}^{\prime \prime}\right\}$ equal respectively to either $\left\{\beta_{N+1}, \gamma_{N+1}\right\}$ or $\left\{\gamma_{N+1}, \alpha_{N+1}\right\}$ or $\left\{\alpha_{N+1}, \beta_{N+1}\right\}$. It is easy to verify then from (11) and the definition of $\sigma_{N}$ that (6) through (10) are satisfied when $N$ is replaced by $N+1$.

Case $\mathrm{B}_{2} . \sigma_{N}$ is either $s_{N, N}^{\prime \prime}+\mathcal{C}\left\{\beta_{N+1}, \gamma_{N+1}\right\}$ or $s_{N, N}^{\prime \prime}+\mathcal{C}\left\{\gamma_{N+1}, \alpha_{N+1}\right\}$ or

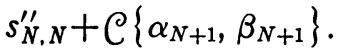

Upon interchanging the superscripts ' and " this reduces to case $B_{1}$.

Case $\mathrm{B}_{3} . \sigma_{N}$ is either $\mathcal{C}\left\{s_{N, N}^{\prime}, s_{N, N}^{\prime \prime}\right\}+\alpha_{N+1}$ or $\mathcal{C}\left\{s_{N, N}^{\prime}, s_{N, N}^{\prime \prime}\right\}+\beta_{N+1}$ or $\mathcal{C}\left\{s_{N, N}^{\prime}, s_{N, N}^{\prime \prime}\right\}+\gamma_{N+1}$.

In this case we set $u(N+1)=u(N) ; \zeta_{n, N+1}^{\prime}=\zeta_{n, N}^{\prime}$ and $\zeta_{n, N+1}^{\prime \prime}=\zeta_{n, N}^{\prime \prime}$ $(n=1,2, \cdots, N)$; and $\zeta_{N+1, N+1}^{\prime}=\zeta_{N+1, N+1}^{\prime \prime}$ equal respectively to $\alpha_{N+1}$ or $\beta_{N+1}$ or $\gamma_{N+1}$.

Again, because of (11) and the definition of $\sigma_{N},(6)$ through (10) hold with $N+1$ replacing $N$.

Thus the proof of Proposition 2 is completed.

2.3. It is worth while to present the two following corollaries, which are of interest in themselves, although we do not need them to establish the main results.

CoROllaRY 1. Under the assumptions of Proposition 1 there exists a sequence $\left(\zeta_{n}\right)_{n=1}^{\infty}$ with $\zeta_{n} \in\left\{\alpha_{n}, \beta_{n}, \gamma_{n}\right\}(n=1,2, \cdots)$ such that

$$
\left|\sum_{\nu=1}^{n} \zeta_{\nu}\right|<5^{1 / 2} \quad(n=1,2, \cdots)
$$

Proof. In the inductive construction used to establish Proposition 2 we had $u(N+1) \geqq u(N)$. Consequently either $u(N) \rightarrow \infty$ or $u(N)=u<\infty$ for all $N \geqq N_{0}$. In the first case $\zeta_{n}=\lim _{N \rightarrow \infty} \zeta_{n, N}^{\prime}$ exists for all $n$. In the second case such a limit exists for $n \neq u$, while for $n=u$ and all sufficiently large $N$, $\zeta_{u, N}^{\prime}$ and $\zeta_{u, N}^{\prime \prime}$ oscillate each over at most two of the numbers $\alpha_{u}, \beta_{u}, \gamma_{u}$; let $\zeta_{u}$ be one of these two numbers. In both cases the sequence $\left(\zeta_{n}\right)_{n=1}^{\infty}$ thus defined satisfies (13).

Specializing by assuming $\gamma_{n}=\beta_{n}$ for all $n$ we have

CoRollary 2. Let $\left\{\alpha_{n}, \beta_{n}\right\}$ be a sequence of pairs of complex numbers such that every one of the segments $\mathcal{C}\left\{\alpha_{n}, \beta_{n}\right\}$ has length not exceeding 2 and contains the origin. Then there exists a sequence $\left(\zeta_{n}\right)_{n=1}^{\infty}$ with each $\zeta_{n}$ equal to either $\alpha_{n}$ or $\beta_{n}$, and such that 


$$
\left|\sum_{\nu=1}^{n} \zeta_{\nu}\right|<5^{1 / 2} \quad(n=1,2, \cdots)
$$

2.4. Using Proposition 1 we now proceed to prove

PROPOSITION 3. Let $\left(\mathrm{Z}_{n}\right)_{n=1}^{\infty}$ be a sequence of uniformly bounded sets of complex numbers such that

$$
0 \in \mathrm{CZ}_{n} \quad(n=1,2, \cdots)
$$

Then $\left(\mathrm{Z}_{n}\right)$ is a convergence factor sequence.

Proof. It is known [6, p. 155] that, because of (14), each $\mathbf{Z}_{n}$ contains three (not necessarily distinct) points $\alpha_{n}, \beta_{n}, \gamma_{n}$ such that $0 \in \mathcal{C}\left\{\alpha_{n}, \beta_{n}, \gamma_{n}\right\} \subset \subset \mathrm{Z}_{n}$ $(n=1,2, \cdots)$. Furthermore, since $\mathbf{Z}_{n} \subset\{|\zeta| \leqq M\}(n=1,2, \cdots)$ we have

$$
\max \left(\left|\gamma_{n}-\beta_{n}\right|,\left|\beta_{n}-\alpha_{n}\right|,\left|\alpha_{n}-\gamma_{n}\right|\right) \leqq 2 M \quad(n=1,2, \cdots) .
$$

Let $\left(a_{n}\right)_{n=1}^{\infty}$ be a given sequence of numbers satisfying (1). We can determine an increasing sequence of integers $\left(N_{k}\right)_{k=1}^{\infty}$ such that $\left|a_{n}\right| \leqq 2^{-k} M^{-1}$ for $n>N_{k}$. Let

$$
\alpha_{n}^{(k)}=2^{k} \alpha_{N_{k}+n} a_{N_{k}+n} ; \quad \beta_{n}^{(k)}=2^{k} \beta_{N_{k}+n} a_{N_{k}+n} ; \quad \gamma_{n}^{(k)}=2^{k} \gamma_{N_{k}+n} a_{N_{k}+n}
$$

$\left(n=1,2, \cdots, N_{k+1}-N_{k} ; k=1,2, \cdots\right)$. Then

$$
0 \in \mathcal{C}\left\{\alpha_{n}^{(k)}, \beta_{n}^{(k)}, \gamma_{n}^{(k)}\right\}
$$

and

$$
\max \left(\left|\gamma_{n}^{(k)}-\beta_{n}^{(k)}\right|,\left|\beta_{n}^{(k)}-\alpha_{n}^{(k)}\right|,\left|\alpha_{n}^{(k)}-\gamma_{n}^{(k)}\right|\right) \leqq 2 .
$$

By Proposition 1 there exist sequences $\left(\zeta_{n}^{(k)}\right)_{n=1}^{N_{k+1}-N_{k}}$ with

$$
\zeta_{n}^{(k)} \in\left\{\alpha_{n}^{(k)}, \beta_{n}^{(k)}, \gamma_{n}^{(k)}\right\}
$$

such that

$$
\left|\sum_{\nu=1}^{n} \zeta_{\nu}^{(k)}\right|<5^{1 / 2} \quad\left(n=1,2, \cdots, N_{k+1}-N_{k} ; k=1,2, \cdots\right) .
$$

We now determine a sequence $\left(\zeta_{n}\right)_{n=1}^{\infty}$ with $\zeta_{n} \in \mathbf{Z}_{n}(n=1,2, \cdots)$ as follows: $\zeta_{n}=\alpha_{n}$ if $n \leqq N_{1}$ or if $a_{n}=0$; while $\zeta_{n}=\left(1 / 2^{k} a_{n}\right) \zeta_{n-N_{k}}^{(k)}$ if $a_{n} \neq 0$ and $N_{k}<n \leqq N_{k+1}$ $(k=1,2, \cdots)$.

Clearly $\zeta_{n} \in\left\{\alpha_{n}, \beta_{n}, \gamma_{n}\right\} \subset \mathrm{Z}_{n}(n=1,2, \cdots)$ and

$$
\left|\sum_{n=N_{k}+1}^{N} \zeta_{n} a_{n}\right|<\frac{5^{1 / 2}}{2^{k}} \quad\left(N=N_{k}+1, N_{k}+2, \cdots, N_{k+1} ; k=1,2, \cdots\right) \text {. }
$$


Hence for any two integers $p, q$ such that $q \geqq p>N_{k}$ we have

$$
\left|\sum_{n=p}^{q} \zeta_{n} a_{n}\right|<2 \frac{5^{1 / 2}}{2^{k}}+\sum_{j=1}^{\infty} \frac{5^{1 / 2}}{2^{k+j}}=\frac{3\left(5^{1 / 2}\right)}{2^{k}}
$$

which, by Cauchy's criterion, establishes the convergence of $\sum_{n=1}^{\infty} \zeta_{n} a_{n}$.

2.5. We can now prove Theorems 1 and 3 . As shown in $\$ 1$ it suffices to prove the latter.

Proof of Theorem 3.

Sufficiency. Let $z_{n} \in \mathcal{C} Z_{n}$ be such that $\left|z_{n}\right|<d_{n}+2^{-n}(n=1,2, \cdots)$ and put

$$
\mathrm{H}_{n}=-z_{n}+\mathrm{Z}_{n} .
$$

Then, if $\sum_{n=1}^{\infty} d_{n}$ converges, the sets $\mathrm{H}_{n}$ are uniformly bounded and $0 \in \mathcal{C} \mathrm{H}_{n}$ $(n=1,2, \cdots)$. By Proposition $3,\left(\mathrm{H}_{n}\right)_{n=1}^{\infty}$ is a convergence factor sequence; thus, given any sequence $\left(a_{n}\right)_{n=1}^{\infty}$ satisfying (1) there exist numbers $\eta_{n} \in \mathrm{H}_{n}$ $(n=1,2, \cdots)$ such that $\sum_{n=1}^{\infty} \eta_{n} a_{n}$ converges.

Upon setting $\zeta_{n}=\eta_{n}+z_{n}(n=1,2, \cdots)$ we have $\zeta_{n} \in Z_{n}$ and, since $\sum_{n=1}^{\infty}\left|z_{n}\right|<\infty$, the series $\sum_{n=1}^{\infty} \zeta_{n} a_{n}$ converges as required.

Necessity. Assume that $\sum_{n=1}^{\infty} d_{n}=\infty$. We shall proceed to construct a sequence $\left(a_{n}\right)_{n=1}^{\infty}$ satisfying (1) such that every series $\sum_{n=1}^{\infty} \zeta_{n} a_{n}$ with $\zeta_{n} \in \mathrm{Z}_{n}$ diverges.

Since $\sum d_{n}$ is divergent there exists a null-sequence $\left(u_{n}\right)_{n=1}^{\infty}$ of real nonnegative numbers such that

$$
\sum_{n=1}^{\infty} d_{n} u_{n}=\infty
$$

and we may assume that $u_{n}=0$ whenever $d_{n}=0$.

Now let $n$ run through all values for which $d_{n} \neq 0$ and denote by $\eta_{n}$ the point of $\overline{\mathrm{CZ}}_{n}$ which is nearest to the origin, then

$$
\operatorname{Re}\left(\zeta \exp \left(-i \arg \zeta_{n}\right)\right) \geqq\left|\eta_{n}\right|=d_{n}>0
$$

for $\zeta \in \overline{\mathrm{CZ}}_{n}$, thus, a fortiori, for all $\zeta \in \mathrm{Z}_{n}$.

The sequence $\left(a_{n}\right)_{n=1}^{\infty}$ defined by $a_{n}=u_{n} \exp \left(-i \arg \eta_{n}\right)$ whenever $d_{n} \neq 0$, and $a_{n}=0$ otherwise, clearly satisfies (1) and, because of (15) and (16), we have, for any choice of $\zeta_{n} \in Z_{n}(n=1,2, \cdots)$,

$$
\sum_{n=1}^{\infty} \operatorname{Re}\left(\zeta_{n} a_{n}\right) \geqq \sum_{n=1}^{\infty} d_{n} u_{n}=\infty
$$

which completes the proof.

3. Sum Factors. The principal results are Theorems 2 and 4 , and, of course, only the latter need be proved.

Proof of Theorem 4. 
3.1. Sufficiency. Let $\left(\mathrm{Z}_{n}\right)_{n=1}^{\infty}$ be a sequence of uniformly bounded sets, that is, there exists a finite, positive $\Delta$ for which

$$
\mathrm{z}_{n} \subset\{|\zeta|<\Delta\} \quad(n=1,2, \cdots),
$$

and assume that there exists a positive number $\delta$ and a positive integer $N_{0}$ such that

$$
\{|\zeta|<\delta\} \subset \mathrm{CZ}_{n} \quad\left(n=N_{0}+1, N_{0}+2, \cdots\right) .
$$

We must prove that given any sequence $\left(a_{n}\right)_{n=1}^{\infty}$ of complex numbers, satisfying (1) and (2), and any number $s$, there exists a sequence $\left(\zeta_{n}\right)_{n=1}^{\infty}$ with $\zeta_{n} \in \mathbf{Z}_{n}(n=1,2, \cdots)$ for which

$$
\sum_{n=1}^{\infty} \zeta_{n} a_{n}=s
$$

holds. This will be done by constructing a sequence $\left(\zeta_{n}\right)$ having the required properties.

Choose $\zeta_{m} \in \mathrm{Z}_{m}$ arbitrarily for $m=1,2, \cdots, N_{0}$ and, having chosen $\zeta_{m} \in \mathbf{Z}_{m}$ for $m=1,2, \cdots, n\left(n \geqq N_{0}\right)$, put $s_{n}=\sum_{m=1}^{n} \zeta_{m} a_{m}$ and choose $\zeta_{n+1}$ $\in \mathrm{Z}_{n+1}$ so that $\left({ }^{3}\right)$

$$
\operatorname{Re}\left(\zeta_{n+1} \exp \left(i\left[\arg a_{n+1}-\arg \left(s-s_{n}\right)\right]\right)>\delta / 2 .\right.
$$

This is clearly possible because of (18). We contend that the sequence thus constructed satisfies (19).

In fact, because of (1) and (2), given any $\epsilon>0$ we can determine $N^{\prime}(\epsilon)$ so large that

$$
\left|a_{n}\right|<\delta \epsilon /\left(2 \Delta^{2}\right) \quad \text { for } n \geqq N^{\prime}(\epsilon)
$$

and then $N^{\prime \prime}(\epsilon)>N^{\prime}(\epsilon)$ so large that

$$
\sum_{n=N^{\prime}(\epsilon)+1}^{N^{\prime \prime}(\epsilon)}\left|a_{n}\right| \geqq \frac{4}{\delta}\left|s-s_{N^{\prime}(\epsilon)}\right| .
$$

Our contention (19) will be established once we prove

$$
\left|s_{n}-s\right| \leqq \epsilon \quad \text { for } n \geqq N^{\prime \prime}(\epsilon) .
$$

Put $\rho_{n}=\left|s-s_{n}\right| ;$ then for $n \geqq N^{\prime}(\epsilon)$ we have

$$
\begin{aligned}
\underset{\rho_{n+1}^{2}}{2}= & \left|s-s_{n}-\zeta_{n+1} a_{n+1}\right|^{2} \\
= & \rho_{n}^{2}-2 \rho_{n}\left|a_{n+1}\right| \operatorname{Re}\left(\zeta_{n+1} \exp i\left[\arg a_{n+1}-\arg \left(s-s_{n}\right)\right]\right) \\
& +\left|\zeta_{n+1}\right|^{2}\left|a_{n+1}\right|^{2}
\end{aligned}
$$

Applying (17) and (20), we obtain

( $\left.{ }^{3}\right)$ For the sake of brevity we make the convention arg $0=0$. 


$$
\rho_{n+1}^{2} \leqq \rho_{n}^{2}-\delta \rho_{n}\left|a_{n+1}\right|+\Delta^{2}\left|a_{n+1}\right|^{2}
$$

If $\rho_{n} \leqq \epsilon$, then, using (21), we have

$$
\begin{aligned}
\underset{\rho_{n+1}}{2} & \leqq\left(\Delta\left|a_{n+1}\right|-\frac{\delta}{2 \Delta} \rho_{n}\right)^{2}+\left(1-\frac{\delta^{2}}{4 \Delta^{2}}\right) \rho_{n}^{2} \\
& \leqq\left(\frac{\delta \epsilon}{2 \Delta}\right)^{2}+\left(1-\frac{\delta^{2}}{4 \Delta^{2}}\right) \epsilon^{2}=\epsilon^{2} .
\end{aligned}
$$

Thus if $\rho_{m} \leqq \epsilon$ for some $m \geqq N^{\prime}(\epsilon)$ then $\rho_{n} \leqq \epsilon$ for all $n>m$.

If $\rho_{n}>\epsilon$ we have, from (21), (25), and $\delta \leqq \Delta$,

$$
\begin{aligned}
\underset{\rho_{n+1}}{2} & \leqq\left(\rho_{n}-\frac{\delta}{4}\left|a_{n+1}\right|\right)^{2}-\left|a_{n+1}\right|\left(\frac{\delta \rho_{n}}{2}-\Delta^{2}\left|a_{n+1}\right|\right)-\frac{\delta^{2}}{16}\left|a_{n+1}\right|^{2} \\
& \leqq\left(\rho_{n}-\frac{\delta}{4}\left|a_{n+1}\right|\right)^{2} .
\end{aligned}
$$

This, combined with the previous result, gives

$$
\rho_{n+1} \leqq \max \left(\epsilon, \rho_{n}-\frac{\delta}{4}\left|a_{n+1}\right|\right) \quad \text { for } n \geqq N^{\prime}(\epsilon) .
$$

By (22) this last inequality implies (23), thus completing the sufficiency part of the proof.

3.2. Necessity. Let $\left(\mathrm{Z}_{n}\right)_{n=1}^{\infty}$ be a sequence of uniformly bounded sets. We assume that, no matter how small $\delta>0$ is taken, there does not exist a value of $N_{0}$ for which (18) holds, and prove that $\left(\mathrm{Z}_{n}\right)_{n=1}^{\infty}$ is not a sum factor sequence.

In fact, by our assumption, there exists an increasing sequence $\left(n_{k}\right)_{k=1}^{\infty}$ of positive integers for which

$$
\left\{|z|<2^{-k}\right\} \nsubseteq C \mathrm{Z}_{n_{k}} \quad(k=1,2, \cdots) .
$$

Consequently there exist real numbers $\vartheta_{k}(k=1,2, \cdots)$ such that

$$
\operatorname{Re}\left(\zeta e^{i \vartheta_{k}}\right)<2^{-k} \quad \text { for } \zeta \in \mathrm{Z}_{n_{k}} \quad(k=1,2, \cdots) .
$$

We now define a sequence $\left(a_{n}\right)_{n=1}^{\infty}$ of numbers as follows:

$$
a_{n_{k}}=\frac{e^{i \vartheta_{k}}}{k}(k=1,2, \cdots) \text { and } a_{n}=0 \text { otherwise. }
$$

Clearly $\left(a_{n}\right)_{n=1}^{\infty}$ satisfies (1) and (2), while for any choice of $\zeta_{n} \in \mathrm{Z}_{n}$ we have, from (26),

$$
\operatorname{Re}\left(\sum_{\nu=1}^{n} \zeta_{\nu} a_{\nu}\right)<\sum_{j=1}^{\infty} \frac{2^{-j}}{j}=\log 2 \quad(n=1,2, \cdots)
$$


Thus, for no choice of $\zeta_{n} \in \mathrm{Z}_{n}$ can we have $\sum_{n=1}^{\infty} \zeta_{n} a_{n}=1$, say, and thus $\left(\mathrm{Z}_{n}\right)$ is not a sum factor sequence.

4. Oscillation of series.

4.1. We first give some general results on the oscillation of series of complex numbers.

A complex number $p$ is called a limit point of a series $\sum_{n=1}^{\infty} a_{n}$ if there exists an increasing sequence of integers $\left(n_{k}\right)_{k=1}^{\infty}$ such that $\left(\sum_{\nu=1}^{n_{k}} a_{v}\right)_{k=1}^{\infty}$ converges to $p$.

The set of limit points of a series is called the derived set of that series.

Definition. $A$ set $D$ is called an oscillation set if there exists a series $\sum_{n=1}^{\infty} a_{n}$ of complex numbers satisfying (1), whose derived set is $D$.

Next we characterize oscillation sets by the following

Proposition 4. A set $D$ is an oscillation set if and only if it is a continuum, or becomes one by adjoining the point at infinity.

By a continuum we understand a nonempty, closed, connected set.

This proposition will follow directly from Proposition 5, which also serves to characterize oscillation sets, but gives more information on the behavior of unbounded subsequences of partial sums. Before stating Proposition 5, some preliminaries are necessary.

Besides the ordinary compactification of the open complex plane II to a sphere by adjoining a point at infinity, we use another compactification of $\Pi$ to a closed disc $\Pi^{*}$. This is done by adjoining to $\Pi$ a circle at infinity whose points are $(\infty, \theta)$ with $\theta$ real mod $2 \pi$. We set $|(\infty, \theta)|=\infty$ and $\arg (\infty, \theta)$ $=\theta$ and define the topology in $\Pi^{*}$ as follows: in the finite part of $\Pi^{*}$ it coincides with that of $\Pi$, while the sets $1 / \epsilon<|z| \leqq \infty ;|\arg z-\theta|<\epsilon$ for all $\epsilon>0$ constitute a fundamental system of neighborhoods of $(\infty, \theta)$.

Definition 4. A set $D^{*}$ in $\Pi^{*}$ is called $a \Pi^{*}$-oscillation set, if there exists a series $\sum_{n=1}^{\infty} a_{n}$ of complex numbers satisfying (1) whose derived set (in the topology of $\Pi^{*}$ ) is $D^{*}$.

The characterization of $\Pi *$-oscillation sets is given by

Proposition 5. A set $D^{*}$ in $\Pi^{*}$ is a $\Pi^{*}$-oscillation set if and only if it is a continuum in $\Pi^{*}$.

If $D^{*}$ is the derived set in $\Pi^{*}$ of a series, then the derived set $D$ of the same series is $D^{*} \cap \Pi$; since $\left.{ }^{4}\right) \Pi^{*}-\Pi$ is connected, it follows that Proposition 5 implies Proposition 4.

(4) The minus sign is used in the set theoretic sense.

As remarked in 1.5 analogous results hold for vectors in Euclidean $n$-space. There is, however, one exception. Proposition 4 does not hold when $n=1$; Proposition 5 is still valid but since $\Pi^{*}-\Pi$, for $n=1$, consists of two points it is not connected and Proposition 4 does not follow. Thus the set $\{x<-1\} \cup\{x>1\}$ which is an oscillation set for complex series is not an oscillation set for real series. 
4.2. Proof of Proposition 5. The derived set $L$ in $\Pi^{*}$ of any series $\sum_{n=1}^{\infty} a_{n}$ is evidently closed and (since $\Pi^{*}$ is compact) nonempty. Assume (1) and suppose that $L$ is not connected. Then there exists a closed set $K$ in $\Pi^{*}$ which separates two points of $L$ and which does not meet $L$. For every open set $K^{\prime} \supset K$, since $a_{n} \rightarrow 0$, there exist infinitely many partial sums which assume values in $K^{\prime}$. Since $\Pi^{*}$, and hence $K$, is compact, these partial sums must have a limit point in $K$, thus contradicting the assumption that $L$ is not connected. Thus $L$ is a continuum.

Conversely suppose a set $D^{*}$ is a continuum in $\Pi^{*}$. We proceed to construct a series $\sum_{n=1}^{\infty} a_{n}$ satisfying (1) whose derived set in $\Pi^{*}$ is $D^{*}$.

Let $\Theta_{n}(n=1,2, \cdots)$ be the set of real numbers $\vartheta$ for which there exist $z$ (in $\Pi^{*}$ ) satisfying

$$
z \in D^{*}, \quad n^{1 / 2} \leqq|z| \leqq \infty, \quad \arg z=\vartheta,
$$

and put

$$
D_{n}=\left(D^{*} \cap\left\{|z|<n^{1 / 2}\right\}\right) \cup\left\{|z|=n^{1 / 2}, \arg z \in \Theta_{n}\right\} .
$$

Then $D_{n}$ is a continuous image of $D^{*}$ by the mapping $\phi_{n}$ defined through $\phi_{n}(z)=z$ for $|z|<n^{1 / 2} ; \phi_{n}(z)=n^{1 / 2} \exp (i \arg z)$ for $|z| \geqq n^{1 / 2}$; hence $D_{n}$ is a continuum.

Let $z^{*}$ be any point of $D^{*}$ and define a sequence $\left(z_{n}\right)_{n=0}^{\infty}$ by $z_{0}=0 ; z_{n}=\phi_{n}\left(z^{*}\right)$ $(n \geqq 1)$.

For each $n=1,2, \cdots$ construct a finite sequence of points $s_{n, 1}, s_{n, 2}, \cdots$, $s_{n, v_{n}}$ satisfying

$$
\begin{array}{lr}
s_{n, 1}=z_{n-1} ; s_{n, r_{n}}=z_{n} ;\left|s_{n, j+1}-s_{n, j}\right|<1 / n^{1 / 2} & \left(j=1,2, \cdots, v_{n}-1\right), \\
s_{n, j} \in D_{n}+\left\{|z|<1 / n^{1 / 2}\right\} & \left(j=1, \cdots, v_{n}\right), \\
D_{n} \subset \bigcup_{j=1}^{v_{n}}\left\{\left|z-s_{n, j}\right|<1 / n^{1 / 2}\right\} .
\end{array}
$$

All the above conditions can be satisfied because each $D_{n}$ is a bounded continuum, $z_{n} \in D_{n}$, and the distance of $z_{n-1}$ from $D_{n}$ is not greater than $\left|z_{n}-z_{n-1}\right| \leqq n^{1 / 2}-(n-1)^{1 / 2} \leqq 1 / n^{1 / 2}$.

Put $m_{k}=\sum_{j=1}^{k} v_{j}, s_{0}=0$ and $s_{n}=s_{k, r}$ for $m_{k}<n=m_{k}+r \leqq m_{k+1}$. Then the sequence $\left(a_{n}\right)_{n=1}^{\infty}$ with $a_{n}=s_{n+1}-s_{n}$ is a null sequence whose partial sums are $s_{n}$ and it is easily verified that $D^{*}$ is the derived set of the series $\sum_{n=1}^{\infty} a_{n}$. q.e.d.

4.3. Let a sum factor sequence $\left(Z_{n}\right)_{n=1}^{\infty}$ of uniformly bounded sets and a series $\sum a_{n}$ satisfying(1) be given and consider the derived sets (in $\Pi$ or in $\left.I^{*}\right)$ of the series $\sum_{n=1}^{\infty} \zeta_{n} a_{n}$ for all possible choices of $\zeta_{n} \in Z_{n}$. We consider the problem of characterizing all these derived sets. Since the sets $\mathbf{Z}_{n}$ are uniformly bounded we clearly have $\zeta_{n} a_{n} \rightarrow 0$ as $n \rightarrow \infty$ and, if $\sum_{n=1}^{\infty}\left|a_{n}\right|<\infty$, also $\sum_{n=1}^{\infty}\left|\zeta_{n} a_{n}\right|<\infty$. Thus if (2) is not satisfied the only derived sets of 
$\sum_{n=1}^{\infty} \zeta_{n} a_{n}$ consist of single points, and the set of points each of which is the limit of the series forms a bounded subset of the plane, depending on $\left(a_{n}\right)_{n=1}^{\infty}$ and $\left(\mathbf{Z}_{n}\right)_{n=1}^{\infty}$, the structure of which may be quite complicated. On the other hand we have already seen (Theorem 4) that if $\left(a_{n}\right)_{n=1}^{\infty}$ satisfies (2) then all sets consisting of a single point are certainly the derived sets of some series $\sum \zeta_{n} a_{n}$. The following theorem shows moreover that if the assumptions of Theorem 4 are satisfied then the $\zeta_{n}$ may be chosen so that any prescribed oscillation set be the derived set of $\sum \zeta_{n} a_{n}$.

THEOREM 5. Let $\left(\mathbf{Z}_{n}\right)_{n=1}^{\infty}$ be a sum factor sequence of uniformly bounded sets. Then, given any sequence $\left(a_{n}\right)_{n=1}^{\infty}$ of complex numbers satisfying (1) and (2) and any continuum $D^{*}$ in $\Pi^{*}$, there exists a sequence $\left(\zeta_{n}\right)_{n=1}^{\infty}$ with $\zeta_{n} \in \mathbf{Z}_{n}$ $(n=1,2, \cdots)$ such that the derived set (in $\left.\Pi^{*}\right)$ of $\sum_{n=1}^{\infty} \zeta_{n} a_{n}$ coincides with $D^{*}$.

Note. Since $Z_{n}$ are uniformly bounded, it follows that $\lim _{n=\infty} \zeta_{n} a_{n}=0$ for any choice of $\zeta_{n} \in Z_{n}$. Therefore, by Proposition 5, this theorem completely characterizes the derived sets of all series of the form $\sum_{n=1}^{\infty} \zeta_{n} a_{n}$.

Proof. By Proposition 5 we can choose a null sequence $\left(a_{n}^{\prime}\right)_{n=1}^{\infty}$ such that the derived set in $\Pi^{*}$ of $\sum_{n=1}^{\infty} a_{n}^{\prime}$ coincides with $D^{*}$. By Theorem 4 there exists an integer $N_{0}$ and two positive numbers $\delta, \Delta$ such that

$$
\{|\zeta| \leqq \delta\} \subset \bigodot \mathrm{Z}_{n} \subset\{|\zeta| \leqq \Delta\} \quad\left(n=N_{0}+1, N_{0}+2, \cdots\right) .
$$

Let $N_{0}$ be the first element of an increasing sequence of integers $\left(N_{k}\right)_{k=0}^{\infty}$ (which we shall define later), choose $\zeta_{m} \in \mathbf{Z}_{m}$ arbitrarily for $m=1,2, \cdots, N_{0}$, and, having chosen $\zeta_{m} \in \mathbf{Z}_{m}$ for $m=1,2, \cdots, n\left(N_{k-1} \leqq n<N_{k}\right)$, put

$$
s_{n}=\sum_{\nu=1}^{n} \zeta_{\nu} a_{\nu} ; \quad s_{k}^{\prime}=\sum_{j=1}^{k} a_{i}^{\prime} \quad \text { and choose } \quad \zeta_{n+1} \in \mathrm{Z}_{n+1}
$$

so that

$$
\begin{aligned}
\operatorname{Re}\left(\zeta_{n+1} \exp \left(i\left[\arg a_{n+1}-\arg \left(s_{k}^{\prime}-s_{n}\right)\right]\right)\right)>\delta / 2 \\
\left(N_{k-1} \leqq n<N_{k}, k=1,2, \cdots\right) .
\end{aligned}
$$

To complete the proof it is necessary only to show that if $\left(N_{k}\right)_{k=1}^{\infty}$ are properly chosen, then $\left(\zeta_{n}\right)_{n=1}^{\infty}$ has the desired property. The choice of $\left(N_{k}\right)$ proceeds as follows: Having chosen $N_{j}$ for $j=0,1,2, \cdots, k-1$, we choose an integer $M_{k}>N_{k-1}$ such that

$$
a_{n}<\frac{\delta\left|a_{k+1}^{\prime}\right|}{2 \Delta^{2}} \quad \text { for } n \geqq M_{k}
$$

and then choose $N_{k}>M_{k}$ so large that

$$
\sum_{n=M_{k}+1}^{N_{k}}\left|a_{n}\right| \geqq \frac{4}{\delta}\left|s_{k}^{\prime}-s_{M_{k}}\right|
$$


On replacing in (29), (30), (31) $s_{k}^{\prime}$ by $s,\left|a_{k+1}^{\prime}\right|$ by $\epsilon, M_{k}$ by $N^{\prime}(\epsilon)$, and $N_{k}$ by $N^{\prime \prime}(\epsilon)$, these inequalities reduce to $(20),(21),(22)$ respectively.

From (23) we have $\left|s_{N_{k}}-s_{k}^{\prime}\right| \leqq a_{k+1}^{\prime}$, and hence $\left|s_{N_{k}}-s_{k+1}^{\prime}\right| \leqq 2\left|a_{k+1}^{\prime}\right|$ $(k=1,2, \cdots)$; while from $(26)$ we get

$$
\left|s_{n}-s_{k+1}^{\prime}\right| \leqq \max \left(2\left|a_{k+1}^{\prime}\right|,\left|a_{k+2}^{\prime}\right|\right) \quad\left(N_{k} \leqq n \leqq N_{k+1}, k=1,2, \cdots\right) \text {. }
$$

This last inequality and $a_{n}^{\prime} \rightarrow 0$ clearly imply that $\sum_{n} \xi_{n} a_{n}$ and $\sum_{k} a_{k}^{\prime}$ have the same derived sets, thus establishing the theorem.

\section{The unbounded case.}

5.1. The set $Z$ in Theorems 1 and 2 was assumed to be bounded, similarly the factor sequences were assumed uniformly bounded in Theorems 3 and 4 . We now proceed to study what happens if the boundedness restrictions are omitted. It turns out that no essentially new convergence factor sets or sequences are added by passing to the unbounded case. The same holds for sum factor sets but for sum factor sequences the situation is considerably more complicated and we do not obtain a characterization of such sequences.

5.2. For convergence factors we have the following two theorems.

Theorem 6. A set $\mathrm{Z}$ of complex numbers is a convergence factor set if and only if it contains a bounded subset which is a convergence factor set.

That is (by Theorem 1), if and only if there exists a bounded set $\mathbf{Z}^{\prime} \subset \mathbf{Z}$ such that $0 \in \overline{\mathrm{CZ}^{\prime}}$.

TheOREm 7. A sequence $\left(\mathrm{Z}_{n}\right)_{n=1}^{\infty}$ of sets of complex numbers is a convergence factor sequence if and only if there exists a sequence of uniformly bounded subsets $\mathrm{Z}_{n}^{\prime} \subset \mathrm{Z}_{n}(n=1,2, \cdots)$ which is itself a convergence factor sequence.

That is (by Theorem 3 ), if and only if there exists a sequence of subsets $\left(\mathrm{Z}_{n}^{\prime}\right)_{n=1}^{\infty}$ satisfying $\sum_{n=1}^{\infty} d_{n}^{\prime}<\infty$ where $d_{n}^{\prime}$ denotes the distance of the origin from $\mathrm{CZ}_{n}^{\prime}$.

Proof. It is enough to establish Theorem 7 and, evidently, only the necessity has to be proved.

We therefore assume that $\left(\mathbf{Z}_{n}\right)_{n=1}^{\infty}$ does not satisfy the conditions of the theorem and show that it cannot be a convergence factor sequence.

Put

$$
\mathrm{Z}_{n}^{(\mathbf{k})}=\mathrm{Z}_{n} \cap\{|\zeta| \leqq k\} \quad(k=1,2, \cdots ; n=1,2, \cdots),
$$

and denote by $d_{n}^{(k)}$ the distance of the origin from $C \mathrm{Z}_{n}^{(k)}$. By assumption $\sum_{n=1}^{\infty} d_{n}^{(k)}=\infty$ for every $k$, therefore there exists an increasing sequence $\left(N_{k}\right)_{k=1}^{\infty}$ of integers (with $N_{0}=0$ ) satisfying

$$
\sum_{n=N_{k}+1}^{N_{k+1}} d_{n}^{(k)} \geqq 1 \quad(k=1,2, \cdots) .
$$


Now put for $k=1,2, \cdots$,

$$
\mathrm{H}_{n}=\mathrm{Z}_{n}^{(k)}, \quad \delta_{n}=d_{n}^{(k)} \quad\left(N_{k}<n \leqq N_{k+1}\right) .
$$

Clearly $\sum \delta_{n}$ is divergent, therefore there exists a null sequence $\left(u_{n}\right)_{n=1}^{\infty}$ of real non-negative numbers such that

$$
\sum_{n=1}^{\infty} \delta_{n} u_{n}=\infty
$$

and we may assume that $u_{n}=0$ whenever $\delta_{n}=0$. Next define a sequence of complex numbers $\left(a_{n}\right)_{n=1}^{\infty}$ through:

Whenever $\delta_{n}=0$ then $a_{n}=0$ and when $\delta_{n} \neq 0$ and $N_{k}<n \leqq N_{k+1}$ then $a_{n}=\max \left(u_{n}, 1 / k\right) \exp \left(-i \arg \eta_{n}\right)$ where $\eta_{n}$ denotes the point of $\overline{\mathcal{C H}}_{n}$ which is nearest to the origin.

The sequence thus constructed satisfies (1) and (2); we claim moreover that for no choice of $\zeta_{n} \in \mathbf{Z}_{n}$ will $\sum \zeta_{n} a_{n}$ be convergent. Indeed, if $\zeta_{n} \in \mathbf{Z}_{n}$ but $\zeta_{n} \notin \mathrm{H}_{n}$ then, by (32), $\left|\zeta_{n}\right| \geqq k$ and thus $\left|\zeta_{n} a_{n}\right| \geqq 1$ unless $a_{n}=0$. Therefore, $\sum \zeta_{n} a_{n}$ cannot converge unless $a_{n} \neq 0$ implies $\zeta_{n} \in \mathrm{H}_{n}$ for all $n$ sufficiently large. But if $\zeta_{n} \in \mathrm{H}_{n}$ and $a_{n} \neq 0$ then we have, cf. (16), $\operatorname{Re}\left(\zeta_{n} a_{n}\right) \geqq \delta_{n} u_{n}$ and thus $\sum \zeta_{n} a_{n}$ is divergent by (33). q.e.d.

5.3. Theorem 2 remains valid if the boundedness condition is dropped.

THEOREM 8. A set $\mathbf{Z}$ (bounded or not) of complex numbers is a sum factor set if and only if 0 is an interior point of $\mathrm{CZ}$.

Indeed, if 0 is an interior point then there exists a subset $\mathbf{Z}^{\prime}$ of three or four points of $\mathbf{Z}$ such that 0 is an interior point of $C Z^{\prime}$. Thus $\mathbf{Z}$ is a sum factor set by Theorem 2. (This shows incidentally that Theorem 8 can be formulated in the same manner as Theorems 6 and 7.)

On the other hand if 0 is not an interior point then there exists a real $\vartheta$ with $\operatorname{Re}\left(\zeta e^{i \vartheta}\right) \leqq 0$ for all $\zeta \in \mathrm{Z}$. Thus taking $a_{n}=e^{i \vartheta} / n$ we see that it is impossible to choose $\zeta_{n} \in \mathrm{Z}$ so that $\sum \zeta_{n} a_{n}$ will converge to a sum with a positive real part.

5.4. The generalization of Theorem 4 to sequences of unbounded or not uniformly bounded sets is not as simple. Any sequence $\left(Z_{n}\right)$ is clearly a sum factor sequence if there exists a sequence of uniformly bounded subsets satisfying the conditions of Theorem 4, that is, which is itself a sum factor sequence. These sufficient conditions are, however, far from being necessary. Thus

$$
\mathrm{Z}_{n}=\{\zeta=0\} \cup\{|\zeta| \geqq n\} \quad(n=1,2, \cdots)
$$

is easily seen to be a sum factor sequence though it fails to satisfy the above conditions. Another example, with each $\mathbf{Z}_{n}$ individually bounded, is furnished by 


$$
\mathrm{Z}_{n}=(\{\operatorname{Re}(\zeta)<0\} \cap\{|\zeta|<n\}) \cup\{|\zeta|=n\} .
$$

The complications of the problem are considerable even if we confine ourselves to real numbers. A sequence $\left(\Xi_{n}\right)_{n=1}^{\infty}$ of sets of real numbers is said to be a sum factor sequence for real series if whenever a real number $s$ and a series of real terms $\sum a_{n}$ satisfying (1) and (2) are given, there exist $\xi_{n} \in \Xi_{n}$ $(n=1,2, \cdots)$ such that $\sum_{n=1}^{\infty} \xi_{n} a_{n}=s$.

From Theorem 3 (or directly by a similar proof) we have:

A sequence of uniformly bounded non-empty sets $\left(\Xi_{n}\right)$ is a sum factor sequence for real series if and only if there exist a positive $\delta$ and an integer $N_{0}$ such that

$$
\{-\delta<\xi<\delta\} \subset \mathcal{C} \Xi_{n} \quad\left(n>N_{0}\right) .
$$

If the boundedness of the sets is retained but not the uniformity, the preceding result has to be modified as follows:

A sequence of bounded sets $\left(\Xi_{n}\right)$ is a sum factor sequence for real series if and only if there exist positive $\delta, \Delta$, and an integer $N_{0}$ satisfying (34),

$$
\{|\xi| \leqq \delta\} \cap \mathcal{C} \Xi_{n} \text { not void } \quad\left(n>N_{0}\right),
$$

and

$$
\sum_{n=1}^{\infty} d_{n}^{\prime}<\infty
$$

where $d_{n}^{\prime}$ denotes the distance of $\xi=0$ from $\mathcal{C}\left(\Xi_{n} \cap\{|\xi| \leqq \Delta\}\right)$.

The significance of (34) is clear, also that of the last condition-it asserts (cf. Theorem 7) that $\left(\Xi_{n}\right)$ is a convergence factor sequence for real series, but (35) is a condition of a new type. If even the boundedness is dropped then the characterization of the above sum sequences is again different. Thus (35) is no longer necessary as is shown by

$$
\Xi_{n}=\{\xi=0\} \cup\{\xi<-n\} \cup\{\xi>n\}, \quad(n=1,2, \cdots) .
$$

\section{An application.}

6.1. To illustrate the way in which the above results may be used we give the following theorem.

THEOREM 9. Let $\left(f_{n}(x)\right)_{n=1}^{\infty}$ be a sequence of nonincreasing positive functions defined for all real $x$ and satisfying, moreover,

$$
\frac{f_{n+1}(y)}{f_{n+1}(x)} \leqq \frac{f_{n}(y)}{f_{n}(x)} \quad \text { whenever } \quad-\infty<x<y<\infty \quad(n=1,2, \cdots) .
$$

Let $\left(c_{n}\right)_{n=1}^{\infty}$ be a sequence of complex numbers and $\left(\mathbf{Z}_{n}\right)_{n=1}^{\infty}$ a sequence of sets satisfying the conditions of Theorem 7. Then it is possible to choose $\zeta_{n} \in \mathbf{Z}_{n}$ $(n=1,2, \cdots)$ so that $\sum_{n=1}^{\infty} \zeta_{n} c_{n} f_{n}(x)$ be convergent for $x>\alpha$ where $\alpha$ is the 
infimum of all $x$ for which

$$
\lim _{n=\infty} c_{n} f_{n}(x)=0 \text {. }
$$

Proof. Let $\left(b_{n}\right)_{n=1}^{\infty}$ be any sequence of complex numbers, then an immediate application of Abel's lemma to (36) gives

$$
\sum_{\nu=p}^{q} b_{\nu} f_{\nu}(y) \leqq \frac{f_{p}(y)}{f_{p}(x)} \max _{p \leqq m \leqq q}\left|\sum_{\nu=p}^{m} b_{\nu} f_{\nu}(x)\right| .
$$

Since the $f_{n}(x)$ are monotone nonincreasing functions this shows that whenever a series of the form $\sum b_{n} f_{n}(x)$ converges for $x=x_{0}$ it does so also for $x>x_{0}$.

We may assume $\alpha<+\infty$ since the contention of the theorem becomes vacuous otherwise. Let $\left(x_{m}\right)_{m=1}^{\infty}$ be a decreasing sequence of (finite) real numbers satisfying $x_{m} \rightarrow \alpha$ as $m \rightarrow \infty$. Clearly $\left(c_{n} f_{n}\left(x_{m}\right)\right)_{n=1}^{\infty}$ are null sequences. By Theorem 7 we can find for every $m$ numbers $\zeta_{n}^{(m)} \in \mathbf{Z}_{n}(n=1,2, \cdots)$ so that the series $\sum_{n} \zeta_{n}^{(m)} c_{n} f_{n}\left(x_{m}\right)$ is convergent $\left(^{5}\right)$. There exists therefore a sequence $\left(N_{m}\right)_{m=1}^{\infty}$ of increasing integers such that

$$
\left|\sum_{n=N_{m}}^{q} \zeta_{n}^{(m)} c_{n} f_{n}\left(x_{m}\right)\right|<2^{-m} \quad(m=1,2, \cdots)
$$

for every $q \geqq N_{m}$. Because of (37) this inequality holds a fortiori also if $x_{m}$ is replaced by any $x>x_{m}$.

We claim that the sequence $\left(\zeta_{n}\right)_{n=1}^{\infty}$ defined by

$$
\zeta_{n}=\zeta_{n}^{(m)} \quad \text { for } N_{m} \leqq n<N_{m+1}(m=1,2, \cdots),
$$

and $\zeta_{n}$ arbitrary (say equal to $\zeta_{n}^{(1)}$ ) for $n<N_{1}$, satisfies the requirement of the theorem. Indeed, if $x>\alpha$ then $z>x_{m}$ for all $m>m_{0}$, and hence we have by (38) for $q>p$ and $N_{m} \leqq p<N_{m+1}$

$$
\left|\sum_{n=p}^{q} \zeta_{n} c_{n} f_{n}(x)\right|<2 \cdot 2^{-m}+2^{-m-1}+2^{-m-2}+\cdots=3 \cdot 2^{-m} .
$$

Thus $\sum \zeta_{n} c_{n} f_{n}(x)$ converges for $x>\alpha$ as required.

6.2. If $\left(\lambda_{n}\right)_{n=1}^{\infty}$ is an increasing sequence of real numbers, the functions $f_{n}(x)=e^{-\lambda_{n} x}$ satisfy the conditions of Theorem 9. Therefore if $\left(\mathrm{Z}_{n}\right)$ is any convergence factor sequence it is possible to choose $\zeta_{n} \in \mathbf{Z}_{n}$ so that the abscissa of convergence of the Dirichlet series $\sum_{n=1}^{\infty} \zeta_{n} c_{n} e^{-\lambda_{n} z}$ coincide with the abscissa of the vanishing of the general term, namely with $\alpha=\lim \sup _{n=\infty}$ $\log \left|c_{n}\right| / \lambda_{n}$. The special case $Z_{n}=\{-1,+1\}$ for all $n$ is contained in [1]; it is used there to obtain the following result:

(5) If $\alpha$ is finite and $\left(c_{n} f_{n}(\alpha)\right)_{n=1}^{\infty}$ is itself a null sequence, the whole diagonal procedure is superfluous. 
Let $\gamma$ denote the abscissa of absolute convergence of $\sum c_{n} e^{-\lambda_{n} z}$, $\left(0<\lambda_{n} \uparrow \infty\right)$. Then given any number $A$ satisfying $\alpha \leqq A \leqq \gamma$ it is possible to choose the \pm signs so that $\sum_{n=1}^{\infty} \pm c_{n} e^{-\lambda_{n} z}$ converges for $\operatorname{Re}(z)>A$ and represents a function having the line $\operatorname{Re}(z)=A$ as a natural boundary.

The proof of this result for $\beta \leqq A<\gamma$ where $\beta$ denotes the abscissa of convergence of $\sum c_{n} e^{-\lambda_{n z}}$ has been sketched in [2]. The extension to $\alpha \leqq A \leqq \gamma$ follows immediately from Theorem 9 .

7. Remark. It is somewhat easier to prove Propositions 1 and 2 if the constant $5^{1 / 2}$ in (5) and (9) is replaced by a larger one. Though any constant would do for the applications we preferred to prove it with $5^{1 / 2}$ since this is quite likely the best possible one. However we cannot prove this. All we can show is that no constant smaller than $\left(2^{2}+2^{-4}\right)^{1 / 2}=(4.0625)^{1 / 2}$ will do; for reasons of brevity the proof of this is omitted.

In the special case of Corollary 2 for which $\alpha_{n}+\beta_{n}=0(n=1,2, \cdots)$ it is known [3] that $5^{1 / 2}$ may be replaced by $3^{1 / 2}$ and Dr. Ch. Hanani and Dr. Th. Motzkin have shown that $3^{1 / 2}$ is the best possible constant in this case.

For vectors in Euclidean spaces of higher dimensions the exact value of the constant is not known even in the special case just mentioned.

\section{BIBLIOGRAPHY}

1. A. Dvoretzky, Studies on general Dirichlet series, Jerusalem, 1941. (Hebrew summary and English abstract of a thesis.)

2. - Sur les changements de signe des coefficients des séries de Dirichlet, C. R. Acad. Sci. Paris vol. 221 (1945) pp. 687-689.

3. A. Dvoretzky and Ch. Hanani, Sur les changements des signes des termes d'une série a termes complexes, C. R. Acad. Sci. Paris vol. 225 (1947) pp. 516-518.

4. H. Hornich, Über Reihen deren Glieder gegen null konvergieren, Monatshefte für Mathematik und Physik vol. 45 (1937) pp. 432-434.

5. — Zu einer geometrischen Theorie der Reihen, Monatshefte für Mathematik und Physik vol. 46 (1938) pp. 266-276.

6. E. Steinitz, Bedingt konvergente Reihen und konvexe Systeme I, J. Reine Angew. Math. vol. 143 (1913) pp. 128-175.

Princeton University,

Princeton, N. J.

HEBREW UNIVERSITY,

JERUSALEM, ISRAEL. 